\title{
Nitrogen regulation of blue light-inducible genes in Neurospora crassa
}

\author{
Vladimir Y. SoKolovsky, ${ }^{1} \uparrow$ Frank-Roman LaUter, ${ }^{1}{ }^{*}{ }^{*}$ Bernd MÜLleR-RöBer, ${ }^{1} \S$ \\ Marta Ricci, ${ }^{1}$ Thomas J. SChmidhauser ${ }^{2}$ and Vincenzo E. A. Russo ${ }^{1}$ \\ ${ }^{1}$ Max-Planck-Institut für Molekulare Genetik, Abteilung Trautner, Ihnestr. 73, 1000 Berlin-Dahlem 33, Germany \\ ${ }^{2}$ Department of Chemistry and Biochemistry, Southern Illinois University Carbondale, Carbondale, Illinois 62901, USA
}

(Received 31 March 1992; revised 16 June 1992; accepted 19 June 1992)

\begin{abstract}
Sexual and asexual differentiation of Neurospora crassa are influenced by nitrogen availability and blue light. Nitrogen limitation induces the production of protoperithecia on solid medium and conidia in liquid medium. Both developmental processes are stimulated by blue light. We have analysed changes in mRNA levels for a variety of light-inducible genes ( $a l, b l i$ and con) under conditions of nitrogen limitation. We show that the photoregulated genes al-1, al-2, bli-4, bli-7, con-5 and con-10 are also regulated by nitrogen limitation. These genes exhibited nitrogen regulation in the nonphotoresponsive mutant strains $w c-1$ and $w c-2$. Therefore, the $w c-1$ and $w c-2$ gene products, although necessary for photoregulation of these al, bli and con genes, are not required for nitrogen regulation.
\end{abstract}

\section{Introduction}

During its life cycle, the heterothallic filamentous fungus Neurospora crassa undergoes asexual and sexual differentiation. Both developmental processes are influenced by external signals, primarily nutrient deprivation and illumination with blue light (Nelson et al., 1975; Turian, 1977; Degli-Innocenti \& Russo, 1984a, $b$ ).

The vegetative growth of the fungus is characterized by the formation of mycelia composed of multinucleate, branched hyphae. In liquid shake cultures, nitrogen limitation activates the developmental process, resulting in production of conidia (Guignard et al., 1984; Müller \& Russo, 1989). These semi-dormant spores represent the final products of asexual differentiation. This process of conidiation involves a series of discrete morphological stages which have been characterized in detail by scanning electron microscopy (Springer \& Yanofsky, 1989). On solid medium, conidiation is induced by desiccation (Nelson et al., 1975) and glucose limitation (Ricci et al., 1991). Once induced, several events in this complex differentiation process are influenced by light, e.g. conidiophores develop towards light (Faull, 1930; Siegel et al., 1968), and illuminated cultures produce

\footnotetext{
* Author for correspondence. fax (415) 7236132 .

† Permanent address: A. N. Bach Institute of Biochemistry, 33 Leninsky Prospekt, Moscow 117071, USSR.

$\ddagger$ Present address: Department of Biological Sciences, Stanford University, Stanford, California 94305-5020, USA.

§ Present address: Institut für Genbiologische Forschung, Ihnestr. 63, 1000 Berlin-Dahlem 33, Germany.
}

conidia faster than dark-grown cultures (Siegel et al., 1968; Turian, 1977).

On solid medium, nitrogen limitation leads to the differentiation of hyphae into the female organs, protoperithecia (Sommer et al., 1987; Ricci et al., 1991). During the sexual process these are fertilized by conidia or vegetative hyphae of the opposite mating type. Once induced by nitrogen deprivation, the formation of protoperithecia is greatly enhanced by blue light (DegliInnocenti et al., 1983; Sommer et al., 1987). In darkgrown mycelia about 10 protoperithecia form per $\mathrm{cm}^{2}$; this increases to about 300 per $\mathrm{cm}^{2}$ after blue light illumination (Sommer et al., 1987).

Recently, two sets of genes have been identified which are of interest with respect to the developmental programs in $N$. crassa. One group, the con genes, was isolated on the basis of preferential expression during conidiation (Berlin \& Yanofsky, 1985). The functions of these genes are unknown. The second group comprises blue-light-inducible (bli) genes; mRNAs corresponding to these genes increase after illumination with blue light (Sommer et al., 1989). The functions of the bli genes, and their expression pattern during conidiation are also unknown. Interestingly, some of the con genes respond to both light and conidiation (Lauter \& Russo, 1991). Two additional genes that are also both light-inducible and expressed during conidiation, albino-1 (al-1) (Schmidhauser et al., 1990; T. J. Schmidhauser, M. S. Sachs \& C. Yanofsky, unpublished data) and albino-2 (al-2) (T. J. Schmidhauser, F.-R. Lauter, V. E. A. Russo \& C. Yanofsky, unpublished data), were used in this analysis. 
The al genes are necessary for carotenoid production (Harding \& Turner, 1981). al-1 encodes the enzyme phytoene dehydrogenase (Schmidhauser et al., 1990) and al-2, phytoene synthase (T. J. Schmidhauser, F.-R. Lauter, V. E. A. Russo \& C. Yanofsky, unpublished results) of $N$. crassa. Although they are developmentally regulated, expression of these genes is not necessary for asexual and sexual development (Degli-Innocenti \& Russo, $1984 a$; Russo, 1986).

Two regulatory mutants of $N$. crassa, wc-1 and $w c-2$, are impaired in light regulation. The products of $w c-1$ and $w c-2$ are required for all photoeffects tested so far (Harding \& Turner, 1981; Harding \& Melles, 1984; Degli-Innocenti \& Russo, 1984a, $b$; Russo, 1988; Sommer et al., 1989; Nelson et al., 1989; Schmidhauser et al., 1990; Nawrath \& Russo, 1990; Lauter \& Russo, 1991), with the exception of photophosphorylation of proteins (Lauter \& Russo, 1990). Wc-1 and Wc-2 are thought to be regulatory factors responsible for the light transduction pathway.

We report here that the light-regulated genes $a l-1, a l-2$, $b l i-4, b l i-7, c o n-5$ and con- 10 are also regulated by nitrogen limitation. Transcript accumulation of those genes is increased upon nitrogen limitation in wild-type as well as $w c-1$ and $w c-2$ mutant strains.

\section{Methods}

Strains and plasmids. N. crassa wild-type (WT) strain (St. Lawrence, STa) was provided by J. R. S. Fincham (Cambridge University, UK). Strains FGSC 4398 ( $w c-1$, a), FGSC $4396(w c-1$, a), FGSC $4408(w c-2$, a) and R251 ( $w c-2$, a) were used as sources of $w c$ RNA. The $w c$ strains were isolated after UV mutagenesis and are isogenic to the WT (STa) (Degli-Innocenti \& Russo, 1984a). The con clones were provided by Charles Yanofsky (Stanford University) (Berlin \& Yanofsky, 1985; Roberts et al., 1988). The $a l-1$ and bli genes were described previously (Schmidhauser et al., 1990; Sommer et al., 1989). In case of al-2, an $800 \mathrm{bp}$ cDNA fragment cloned in pTJS430 was used as hybridization probe.

Growth of $N$. crassa in liquid culture medium. $N$. crassa mycelia were grown in liquid culture, with shaking, as described by Chambers $e t$ al. (1985). Mycelia were grown in darkness in $75 \mathrm{ml}$ medium in $250 \mathrm{ml}$ flasks at $34{ }^{\circ} \mathrm{C}$ for $24 \mathrm{~h}$ prior to harvest. Modified Vogel's minimal medium (Russo, 1988) supplemented with $2 \%(\mathrm{w} / \mathrm{v})$ glucose and $50 \mathrm{mM}-\mathrm{NH}_{4} \mathrm{Cl}$ as carbon and nitrogen sources, respectively, was used for cultivation. In nitrogen limitation experiments, mycelia were grown in the dark for $24 \mathrm{~h}$ in a medium supplemented with $2 \mathrm{mM}-\mathrm{NH}_{4} \mathrm{Cl}$. Mycelia were harvested under red safety light by filtration (Chambers et al., 1985) and immediately frozen in liquid nitrogen.

Culture of $N$. crassa on solid medium. $N$. crassa was grown in $8 \mathrm{~cm}$ diameter Petri dishes containing $20 \mathrm{ml}$ agar medium. The agar medium was modified Vogel's minimal medium (Russo, 1988) supplemented with $1.5 \%(\mathrm{w} / \mathrm{v})$ Bacto-Agar (Difco), with $1 \%(\mathrm{w} / \mathrm{v})$ L-sorbose, and $0.1 \%$ glucose as carbon sources, with $4 \mathrm{mM}-\mathrm{NH}_{4} \mathrm{Cl}$ as the sole nitrogen source. Solid medium was covered with a circular dialysis membrane (Visking, Serva). For inoculation, $10^{4}$ conidia per plate were spread onto the dialysis membrane. Mycelia were grown in darkness at $23^{\circ} \mathrm{C}$. After $3 \mathrm{~d}$, membranes overgrown with mycelia were transferred onto new plates that contained zero or $50 \mathrm{mM}-\mathrm{NH}_{4} \mathrm{Cl}$ as sole nitrogen source. After $24 \mathrm{~h}$ additional growth in darkness at $23^{\circ} \mathrm{C}$, cells were harvested by scraping them from the upper surface of the dialysis membranes. All manipulations were performed under a red safety light. Collected mycelia were immediately frozen in liquid nitrogen.

RNA dot blot analysis. Extraction of total RNA was performed as described previously (Sokolovsky et al., 1990), an extraction method developed for RNAase-rich cultures. RNA dot-blots were performed as described by Boll et al. (1986). Radioactively labelled DNA probes were prepared using random primer labelling technique (Feinberg \& Vogelstein, 1983). Quantification of mRNA was done as described previously (Sommer et al., 1989)

\section{Results}

\section{Nitrogen regulation of light-inducible genes in mycelia grown in liquid and on solid medium}

We wished to determine the expression patterns of lightregulated genes in response to nitrogen availability, a stimulus known to influence development. Nitrogen limitation induces conidiation in liquid media (Guignard et al., 1984, Müller \& Russo, 1989), and both inhibits conidiation (Ricci et al., 1991) and induces protoperithecia formation (Sommer et al., 1987; Ricci et al., 1991) on solid media. Since different developmental processes may be induced by the same stimulus, depending on growth conditions, we have compared the effects of nitrogen limitation on the transcription patterns of photoregulated genes in mycelia grown in liquid culture and on solid agar. Cultures were grown in darkness under conditions of low or high nitrogen supply, and the amount of mRNA expressed from different genes was measured by dot-blot analysis (Boll et al., 1986). Our results, based on RNA extracted from three to five different mycelial cultures grown in liquid medium and two different mycelial cultures grown on solid medium, are compared in Table 1.

Under conditions of nitrogen limitation, mRNA levels for all the photoregulated genes except $b l i-3$ increased by more than a factor of 3 in wild-type cultures grown in liquid medium. The slight increase in transcript levels of bli-3 was not considered significant. A greater than 10fold increase in accumulation was observed for the al-1 and $b l i-7$ RNAs. The increase in mRNA level for $b l i-7$, a gene which is weakly photoregulated (Sommer et al., 1989), was appreciable; a greater than 50-fold accumulation was observed. In the induced state, the bli-7 mRNA represented about $0.25 \%$ of the total RNA (Table 1). Under conditions of nitrogen limitation, the variation in bli-7 transcript levels among different experiments was fairly low (less than $10 \%$ ). However, fluctuation of bli-7 transcript levels has been observed in mycelia grown in complete medium (Tables 1 and 2; 
Table 1. Gene regulation in response to nitrogen limitation using liquid and solid growth media

Total RNA was isolated from dark-grown wild-type (STa) $N$. crassa cultures, with low $(\mathrm{N}-)$ or high $(\mathrm{N}+)$ nitrogen supply (see Methods). mRNA accumulation from seven photoinducible (top group) and two photoindependent (lower group) genes was monitored by dot-blot analysis. See Methods for mRNA quantification. Data are means from 3-5 independent RNA extractions $( \pm$ SEM) for liquid cultures; for solid cultures, two values ( $a$, first experiment; $b$, second experiment) are quoted.

\begin{tabular}{|c|c|c|c|c|}
\hline \multirow[b]{4}{*}{ Gene } & \multicolumn{4}{|c|}{ 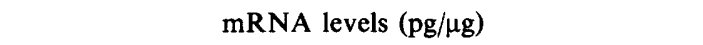 } \\
\hline & \multicolumn{2}{|c|}{ Liquid medium } & \multicolumn{2}{|c|}{ Solid medium } \\
\hline & $N+$ & $\mathbf{N}-$ & $\mathbf{N}+$ & $N-$ \\
\hline & & & $a / b$ & $a / b$ \\
\hline$a l-1$ & $4 \cdot 5( \pm 1 \cdot 6)$ & $63 \cdot 7( \pm 5 \cdot 8)$ & $3 \cdot 5 / 11$ & $13 / 120$ \\
\hline$a l-2$ & $1.2( \pm 0.4)$ & $8 \cdot 6( \pm 2 \cdot 0)$ & $1 \cdot 8 / 1 \cdot 8$ & $12 / 20$ \\
\hline bli-3 & $11 \cdot 4( \pm 3 \cdot 1)$ & $14.7( \pm 0.8)$ & $14 / 29$ & $15 / 43$ \\
\hline bli-4 & $1.2( \pm 0.3)$ & $4.1( \pm 0.7)$ & $3 / 5$ & $9 / 26$ \\
\hline bli-7 & $52 \cdot 1( \pm 21 \cdot 7)$ & $2699 \cdot 2( \pm 256 \cdot 2)$ & $19 / 142$ & $27 / 67$ \\
\hline con-5 & $11 \cdot 4( \pm 3 \cdot 2)$ & $47 \cdot 2( \pm 4 \cdot 5)$ & $10 / 27$ & $21 / 130$ \\
\hline con- 10 & $0.6( \pm 0.2)$ & $2.7( \pm 0.8)$ & $0 \cdot 5 / 10$ & $0 \cdot 5 / 4$ \\
\hline$a m$ & $11.9( \pm 2 \cdot 4)$ & $190 \cdot 0( \pm 16 \cdot 7)$ & $75 / 47$ & $88 / 59$ \\
\hline$t u b-2$ & $3 \cdot 7( \pm 0 \cdot 4)$ & $8.5( \pm 2.0)$ & $22 / 19$ & $44 / 41$ \\
\hline
\end{tabular}

Sommer et al., 1989). Two nonphotoregulated genes; tub-2, which encodes $\beta$-tubulin, a subunit of cytoskeleton microtubules (Orbach et al., 1986), and am, which encodes NADP-specific glutamate dehydrogenase (GDH) of $N$. crassa were included for comparison. GDH is an enzyme of the nitrogen assimilatory pathway and converts ammonia to glutamate (Kinnard et al., 1982). Expression of $t u b-2$ was slightly increased under conditions of nitrogen limitation. Transcription of am was under nitrogen control in liquid medium (Table 1); an induction factor greater than 10 was observed.

In two independent experiments, nitrogen regulation was also analysed in mycelia grown on solid agar. As in liquid medium, transcript levels of $a l-2$ and bli-4 increased under conditions of nitrogen limitation (Table 1). The induction factors were comparably high under both growth conditions. In case of $a l-1, c o n-5$ and con-10, the variation between the two experiments was too high to infer whether or not the mRNA levels of those genes increased under conditions of nitrogen limitation. As in liquid medium, transcript levels of $t u b-2$ increased slightly in cultures shifted to a nitrogen-free medium; the slight increase in mRNA levels of bli-3 was not considered significant. Interestingly, transcription of am and bli-7, although strongly nitrogen-regulated in mycelia grown in liquid cultures, were uninducible by nitrogen limitation in mycelia grown on solid medium. This effect was especially significant in the case of bli-7. Under conditions of nitrogen limitation, bli-7 mRNA, measured in cultures grown on solid agar medium, represented only $1-2 \%$ of the amount measured in cultures grown in liquid medium.

\section{Role of wc genes on nitrogen induced gene expression}

Nitrogen regulation of photoinducible genes was also examined in $w c$ strains. The $w c$ mutants are blind for most of the photoeffects tested so far. Transcripts of the photoregulated al (Schmidhauser et al., 1990; Nelson et al., 1989; F.-R. Lauter, T. J. Schmidhauser, C. Yanofsky \& V. E. A. Russo, unpublished results), bli (Sommer $e t$ $a l ., 1989)$ and con genes (Lauter \& Russo, 1991) do not increase after light stimulation in $w c-1$ and $w c-2$ strains.

In order to study nitrogen regulation in these mutants, the fungus was grown in darkness in liquid medium with low or high nitrogen supply. Two alleles each of $w c-1$ and $w c-2$ were used. Results are presented in Table 2 . The response of al-1, al-2, bli-3, bli-4, bli-7 and tub-2 to nitrogen limitation was similar to WT in the $w c$ mutant strains. RNA accumulation for con-5, con- 10 and am was slightly altered in $w c$ strains following nitrogen limitation relative to wild-type, but still nitrogen-regulated. The products of the $w c-1$ and $w c-2$ genes do not appear to be required for nitrogen regulation of the genes analysed in these studies.

\section{Discussion}

Sexual as well as asexual differentiation of $N$. crassa is induced by nitrogen limitation (Nelson et al., 1975; Guignard et al., 1984; Sommer et al., 1987; Müller \& Russo, 1989; Ricci et al., 1991). Both developmental processes are also influenced by light (Faull, 1930; Sargent et al., 1966; Sargent \& Briggs, 1967; Siegel et al., 1968; Turian, 1977; Degli-Innocenti et al., 1983; Sommer et al., 1987). We have analysed transcript levels of blue-light-inducible genes under conditions of nitrogen limitation. In cultures grown in liquid medium the amounts of al-1, al-2, bli-4, bli-7, con-5 and con-10 mRNAs were also regulated by nitrogen availability in the medium. Therefore, these genes are under dual environmental control, being regulated by either nitrogen or light.

In addition, two non-photoregulated genes, $t u b-2$ and $a m$, were analysed. $t u b-2$ RNA levels were independent of the nitrogen supply, but am transcripts increased by a factor of more than 10 when nitrogen was limiting. NADP-specific GDH activity (Dantzig et al., 1979) and am mRNA levels are known to be under nitrogen control (Frederick \& Kinsey, 1990).

The influence of a third environmental parameter, liquid versus solid growth media, was also analysed. In the laboratory, $N$. crassa is normally grown either in 
Table 2. Gene regulation under nitrogen limitation: wild-type vs. wc-1 and wc-2 mutant strains

Total RNA was isolated from $N$. crassa cultures dark-grown in liquid medium with high or low nitrogen supply (see Methods). Accumulation of specified trancripts was monitored by dot-blot analysis and quantified by densitometry. Values are means ( \pm SEM) from 3-5 independent extractions per growth condition and strain; ratios were calculated for each high/low $\mathrm{N}$ pair and mean ratios then calculated. All data were normalized to $n-6$ (Sommer et al., 1989), a nitrogen-independent gene (not shown). Two alleles of each $w c$ locus were used. R251 is a wc-2 strain isolated in the Russo laboratory after UV mutagenesis, isogenic to the wild-type STa.

\begin{tabular}{|c|c|c|c|c|c|}
\hline \multirow[b]{3}{*}{ Gene } & \multicolumn{5}{|c|}{ Relative mRNA levels $\mathrm{N}-/ \mathrm{N}+$} \\
\hline & & $w c-1$ & $w c-1$ & $w c-2$ & $w c-2$ \\
\hline & WT & 4398 & 4396 & 4408 & $\mathrm{R} 251$ \\
\hline$a l-1$ & $20 \cdot 8( \pm 5 \cdot 8)$ & $25 \cdot 6( \pm 7 \cdot 8)$ & $35 \cdot 1( \pm 7 \cdot 4)$ & $48 \cdot 3( \pm 15 \cdot 0)$ & $20 \cdot 0( \pm 9 \cdot 5)$ \\
\hline$a l-2$ & $7 \cdot 5( \pm 2 \cdot 6)$ & $5.1( \pm 0.8)$ & $12 \cdot 1( \pm 2 \cdot 7)$ & $9.3( \pm 3.9)$ & $7 \cdot 1( \pm 1 \cdot 2)$ \\
\hline$b l i-3$ & $1.9( \pm 0.7)$ & $1.9( \pm 0.8)$ & $1 \cdot 1( \pm 0.5)$ & $1.8( \pm 0.7)$ & $1 \cdot 1( \pm 0 \cdot 3)$ \\
\hline bli-4 & $4 \cdot 1( \pm 0.9)$ & $2 \cdot 5( \pm 0.5)$ & $5.0( \pm 1 \cdot 1)$ & $3.7( \pm 0.8)$ & $2.3( \pm 0.6)$ \\
\hline bli-7 & $176.5( \pm 87.2)$ & $80.4( \pm 23.4)$ & $153.0( \pm 91 \cdot 3)$ & $85.0( \pm 19 \cdot 1)$ & $65.0( \pm 24.6)$ \\
\hline con-5 & $4 \cdot 1( \pm 0 \cdot 4)$ & $2.0( \pm 0.4)$ & $3.0( \pm 0.2)$ & $2.0( \pm 0.4)$ & $1.8( \pm 0.6)$ \\
\hline con- 10 & $4 \cdot 2( \pm 1 \cdot 2)$ & $8 \cdot 7( \pm 1 \cdot 3)$ & $9 \cdot 2( \pm 1 \cdot 1)$ & $7 \cdot 7( \pm 1 \cdot 1)$ & $6 \cdot 1( \pm 2 \cdot 9)$ \\
\hline$a m$ & $17 \cdot 8( \pm 4 \cdot 0)$ & $5 \cdot 1( \pm 0 \cdot 8)$ & $20 \cdot 1( \pm 7 \cdot 3)$ & $5 \cdot 8( \pm 1 \cdot 6)$ & $7 \cdot 3( \pm 2 \cdot 6)$ \\
\hline$t u b-2$ & $2 \cdot 1( \pm 0.6)$ & $1.0( \pm 0.4)$ & $1.9( \pm 0.5)$ & $1 \cdot 3( \pm 0 \cdot 3)$ & $1 \cdot 3( \pm 0.2)$ \\
\hline
\end{tabular}

WT, wild-type.

shaking liquid medium, submerged, or on solid agar. In liquid medium, nitrogen and glucose starvation leads to conidiation (Guignard et al., 1984; Müller \& Russo, 1989), and no protoperithecia. On solid agar, nitrogen limitation induces protoperithecia formation (Sommer $e t$ al., 1987) and suppresses conidiation, while glucose limitation induces conidiation and suppresses protoperithecia formation (Ricci et al., 1991). In our experiments, despite their limited number and the variation between them, it was apparent that the expression of bli-7 and am differed significantly in cultures grown in liquid and on solid medium.

RNA levels for bli-7 are strongly nitrogen-regulated in liquid medium but unregulated on solid agar. This implies that bli-7 is regulated by at least three parameters; light, nitrogen availability, and the method of cultivation (liquid versus solid medium). Gene inactivation experiments should show whether the changes in bli-7 mRNA levels were accidentally parallel to the morphological transformations associated with conidiation or whether the product of this gene is essential for normal asexual differentiation. The amount of bli-7 mRNA in cells grown in liquid medium under conditions of nitrogen limitation was $0.25 \%$ of the total RNA. Assuming that $b l i-7$ mRNA is polyadenylated, that poly $\left(\mathrm{A}^{+}\right)$mRNA represents $1-2 \%$ of the total RNA of $N$. crassa (Sachs \& Yanofsky, 1991) and that the relative amounts of rRNA and tRNA do not change during nitrogen starvation, the amount of bli-7 mRNA can be estimated to be $10-25 \%$ of the total poly $\left(\mathrm{A}^{+}\right)$mRNA mass.

Why is bli-7 nitrogen-regulated in $N$. crassa grown in liquid but not on solid medium? One important difference between liquid and solid medium is the amounts of $\mathrm{O}_{2}$ and $\mathrm{CO}_{2}$ that are available to the organism. It is known that $\mathrm{CO}_{2}$ inhibits conidiation in $N$. crassa (Charles, 1962; Sargent \& Kaldenborn, 1972) and differentiation of the fungus Phycomyces (Russo et al., 1981).

How is gene regulation by light and nitrogen achieved in $N$. crassa? Which elements participate in these regulatory processes? Two genes, $w c-1$ and $w c-2$, are likely to play an important role in the blue-light signal transduction chain of $N$. crassa (Harding \& Turner, 1981; Degli-Innocenti \& Russo, 1984b) and to influence proper dephosphorylation of proteins (Lauter \& Russo, 1990). Their gene products are necessary for photocarotenogenesis (Harding \& Turner, 1981), phototropism of perithecial beaks (Harding \& Melles, 1984), photoinduction of protoperithecia (Degli-Innocenti \& Russo, 1984a) and photoinduction of circadian rhythms in $b d$ mutants (Russo, 1988). In mycelia of $w c-1$ and $w c-2$ mutant strains, transcription of $a l, b l i$ and con genes (Sommer $e t$ al., 1989; Nelson et al., 1989; Schmidhauser et al., 1990; Lauter \& Russo, 1991) is not photoinducible.

The results presented here show that nitrogen regulation of al-1, al-2, bli-4, bli-7, con-5 and con-10 was not dramatically altered in $w c$ mutant strains. Therefore, the products of $w c-1$ and $w c-2$, while absolutely necessary for light regulation of al, bli and con genes, are not needed for their nitrogen regulation.

RNA accumulation of $a l-1, a l-2, b l i-4, b l i-7$, con-5 and con-10 is regulated by two environmental factors that also influence development of $N$. crassa: nitrogen availability and blue light. Gene inactivation experiments should show whether some of the bli or con genes 
are needed for sexual or asexual differentiation processes in this fungus. Deletion analyses and study of proteinDNA interactions should lead to the identification of $c i s$ and trans acting elements that are involved in dual environmental control of those genes.

We appreciate the critical comments of Charles Yanofsky, Aijth Kamath, Matthew Springer and Carl Yamashiro. We would like to thank Matthew Sachs and Karl Hager for providing con DNAs. F. R. L. was supported by the Deutsche Forschungsgemeinschaft (Ru 206/4-1 and La 714/1-1). V.Y.S. and F. R.L. contributed equally to this work.

\section{References}

BERLIN, V. \& YANOFSKY, C. (1985). Isolation and characterization of genes differentially expressed during conidiation of Neurospora crassa. Molecular and Cellular Biology 5, 849-855.

Boll, W., Fujisawa, J.-I., Niemi, J. \& Weismann, C. (1986). A new approach to high sensitivity differential hybridization. Gene 50, 4153.

Chambers, J. A. A., Hinkelammert, K. \& Russo, V. E. A. (1985). Light-regulated protein and poly(A) ${ }^{+}$mRNA synthesis in Neurospora crassa. EMBO Journal 4, 3649-3653.

CharLes, H. P. (1962). Response of Neurospora mutants to carbon dioxide. Nature, London 195, 359-360.

Dantzig, A. H., WiegmanN, JR, F-L. \& Nason, A. (1979). Regulation of the glutamate dehydrogenase in nit-2 and am mutants of Neurospora crassa. Journal of Bacteriology 137, 1333-1339.

Degli-InNocenti, F., PoHL, U. \& Russo, V. E. A. (1983). Photoinduction of protoperithecia in Neurospora crassa by blue light. Photochemistry and Photobiology 37, 49-51.

DEGLI-INNOCENTI, F. \& Russo, V. E. A. (1984a). Isolation of new white collar mutants of Neurospora crassa and studies on their behavior in the blue-light-induced formation of protoperithecia. Journal of Bacteriology 159, 757-761.

Degli-InNocenti, F. \& Russo, V. E. A. (1984b). Genetic analysis of blue-light-induced responces in Neurospora crassa. In Blue Light Effects in Biological Systems, pp. 214-219. Edited by H. Senger. Berlin: Springer Verlag.

FAULL, A. F. (1930). On the resistance of Neurospora crassa. Mycologia 22, 288-303.

Feinberg, A. P. \& Vogelstein, B. (1983). A technique for radiolabeling DNA restriction endonuclease fragments to high specific activity. Analytical Biochemistry 132, 6-13.

FrEDERICK, G. D. \& KinseY, J. A. (1990). Distant upstream regulatory sequences control the level of expression of the am (GDH) locus of Neurospora crassa. Current Genetics 18, 53-58.

Guignard, R., Grange, F. \& Turian, G. (1984). Microcycle conidiation induced by partial nitrogen deprivation in Neurospora crassa. Canadian Journal of Microbiology 30, 1210-1215.

HaRding, R. W. \& Melles, S. (1984). Genetic analysis of the phototropism of Neurospora crassa perithecial beaks using white collar and albino mutants. Plant Physiology 72, 996-1000.

Harding, R. W. \& TURner, R. V. (1981). Photoregulation of the carotenoid biosynthetic pathway in albino and white collar mutants of Neurospora crassa. Plant Physiology 68, 745-749.

Kinnaird, J. H., Keighren, M. A., Kinsey, J. A., Eaton, M. \& FINCHAM, J. R. S. (1982). Cloning of the am (glutamate dehydrogenase) gene of Neurospora crassa through the use of a synthetic DNA probe. Gene 20, 387-396.

LAUTER, F.-R. \& RUSSo, V. E. A. (1990). Light-induced dephosphorylation of a $33 \mathrm{kDa}$ protein in the wild-type strain of Neurospora crassa the regulatory mutants $w c-1$ and $w c-2$ are abnormal. Journal of Photochemistry and Photobiology 5, 95-103.

LAUTER, F.-R. \& Russo, V. E. A. (1991). Blue light induction of conidiation-specific genes in Neurospora crassa. Nucleic Acids Research 19, 6883-6886.
MülleR, B. \& Russo V. E. A. (1989). Nitrogen starvation or glucose limitation induces conidiation in constantly shaken liquid cultures of Neurospora crassa. Fungal Genetics Newsletters 36, 58-60.

Nawrath, C. \& Russo, V. E. A. (1990). Fast induction of translatable mRNA by blue light in Neurospora crassa wildtype: the $w c-1$ and $w c-$ 2 mutants are blind. Journal of Photochemistry and Photobiology 4, 261-271.

Nelson, M. A., Morelli, G., Carattoli, A., Romano, N. \& Macino, G. (1989). Molecular cloning of a Neurospora crassa carotenoid biosynthetic gene (albino-3) regulated by blue light and the products of the white collar genes. Molecular and Cellular Biology 9, 12711276.

Nelson, R. E., Selitrennixoff, C. P. \& Siegel, R. W., (1975) Results and Problems in Cell Differentiation, pp. 291-310. Edited by J. Reinert and H. Holzer. Berlin: Springer.

Orbach, M. J., Porro, E. B. AND Yanofsky, C. (1986). Cloning and characterization of the gene for $\beta$-tubulin from a benomyl-resistant mutant of Neurospora crassa and its use as a dominant selectable marker. Molecular and Cellular Biology 6, 2452-2461.

RicCi, M., KrappmanN, D. \& Russo, V. E. A. (1991). Nitrogen and carbon starvation regulate conidiation and protoperithecia formation in Neurospora crassa grown on solid medium. Fungal Genetics Newsletters 38, 87-88.

Roberts, A. N., Berlin, V., Hager, K. \& Yanofsky, C. (1988). Molecular analysis of a Neurospora crassa gene expressed during conidiation. Molecular and Cellular Biology 8, 2411-2418.

Russo, V. E. A. (1986) Are carotinoids the blue light photoreceptor in the photoinduction of protoperithecia in Neurospora crassa? Planta $168,56-60$.

Russo, V. E. A. (1988). Blue light induces circadian rhythms in the $b d$ mutant of Neurospora: double mutants $b d, w c-l$ and $b d, w c-2$ are blind. Journal of Photochemistry and Photobiology 2, 59-65.

Russo, V. E. A., POHL, U. \& VolPI, L. (1981). Carbon dioxide inhibits photogenesis in Phycomyces and blue light overcomes this inhibition. Photochemistry and Photobiology 34, 233-236.

SACHS, M. S. \& YANOFSKY, C. (1991). Developmental expression of genes involved in conidiation and aminoacid starvation in Neurospora crassa. Developmental Biology 148, 117-128.

SARGENT, M. L. \& BRIGGS, W. R. (1967). The effects of light on a circadian rhythm of conidiation in Neurospora. Plant Physiology 42, 1504-1510.

SARGENT, M. L. \& KaLdenborn. (1972). Effects of medium composition and carbon dioxide on circadian conidiation in Neurospora. Plant Physiology 50, 171-175.

SARgent, M. L., Briggs, W. R. \& WoOdWard, D. O. (1966). Circadian nature of a rhythm expressed by an invertaseless strain of Neurospora crassa. Plant Physiology 41, 1343-1349.

SCHMidhauser, T. J., LaUter, F.-R., Russo, V. E. A. \& Yanofsky, C. (1990). Cloning, sequence, and photoregulation of $a l-1$, a carotenoid biosynthetic gene of Neurospora crassa. Molecular and Cellular Biology 10, 5064-5070.

Siegel, R. W., Matsuyama, S. S. \& Urey, J. C. (1968). Induced macroconidiation formation in Neurospora crassa. Experientia 24, $1179-1181$.

Sokolovsky, V. Y., Kaldenhoff, R., Ricci, M. \& Russo, V. E. A. (1990). Fast and reliable mini-prep RNA extraction from Neurospora crassa. Fungal Genetics Newsletters 37, 39-40.

Sommer, T., Degli Innocenti, F. \& Russo, V. E. A. (1987). Role of nitrogen in the photoinduction of protoperithecia and carotenoids in Neurospora crassa. Planta 170, 205-208.

Sommer, T., Chambers, J. A. A., Eberle, J., Lauter, F.-R. \& Russo, V. E. A. (1989). Fast light-regulated genes Neurospora crassa. Nucleic Acids Research 17, 5713-5723.

SPRINGER, M. L. \& YANOFSKY, C. (1989). A morphological and genetic analysis of the conidiophore development in Neurospora crassa. Genes and Development 3, 559-571.

TURIAN, G. (1977). Fungal differentiation. In Biotechnology and Fungal Differentiation, pp. 1-15. Edited by J. Meyrath \& J. D. Bu'Lock. London: Academic Press. 